\title{
Como afecta la exposición aérea el equilibrio ácido base de organismos móviles del intermareal: Petrolisthes laevigatus (Guérin, 1835) (Decapoda: Porcellanidae), como caso de estudio
}

\author{
Effect of aerial exposure in acid-base equilibrium in intertidal mobile organisms: Petrolisthes \\ laevigatus (Guérin, 1835) (Decapoda: Porcellanidae) as study case \\ Marcelo E. $\operatorname{Lagos}^{1}$ y Cristian W. Cáceres ${ }^{1}$ \\ ${ }^{1}$ Departamento de Ecología Costera, Facultad de Ciencias, Universidad Católica de la Santísima Concepción, \\ Casilla 297, Concepción, Chile \\ ccaceres@ucsc.cl
}

\begin{abstract}
Due to the action of tidal rhythms, intertidal animals can spend $50 \%$ of their lives exposed to aerial conditions, which produces highly specific physiological responses (e.g. high tolerance to desiccation, development of alternative pathways of oxygen uptake). Such responses have mostly been studied in sessile species. In this article, we studied physiological responses in the concentrations of calcium and $\mathrm{CO}_{2}$ partial pressure in the hemolymph of the intertidal crab Petrolisthes laevigatus. We experimentally exposed individuals of this species to variations in aerial exposure $(0,6,15,60$, $120,240$ and $360 \mathrm{~min})$. Changes in $\mathrm{pH}$, concentrations of calcium $\left(\mathrm{Ca}^{2+}\right)$ in the hemolymph and exoskeleton, bicarbonate ion $\left(\mathrm{HCO}_{3}\right)$ and the partial pressure of $\mathrm{CO} 2$ in the hemolymph were evaluated. The results show a reduction of exoskeleton calcium $\left[\mathrm{Ca}^{2+}\right]$ and an increase in hemolymphatic calcium $\left[\mathrm{Ca}^{2+}\right]$ concentrations with increasing aerial exposition. These results could indicate the dissolution of exoskeleton $\mathrm{CaCO}_{3}$, suggesting a regulation of the acid-base balance during aerial exposure. The lowest $\mathrm{pH}$ and the highest $\mathrm{HCO}_{3}$ - and $\mathrm{PCO}_{2}$ concentrations were found in the group with 0 minutes of aerial exposure (i.e. submerged conditions). This results would indicate a respiratory alkalosis response during emersion periods, which results in reduced breathing capacity during periods of immersion.
\end{abstract}

Key words: Crab, homeostasis, physiological adaptation, respiration

\section{Introducción}

El hábitat intermareal es altamente productivo, con una gran diversidad y variable en espacio y tiempo. Los cambios regulares y periódicos en el nivel del mar durante los ciclos mareales, generan exposición aérea a los organismos que habitan en esta franja (Martin \& Bridges 1999). En zonas costeras poco profundas las variaciones ambientales son además influenciadas por otros procesos naturales (e.g. cursos de agua dulce) y por efectos
Resumen.- Por acción del ritmo de las mareas, los organismos intermareales pueden pasar alrededor del $50 \%$ de sus vidas expuestos a condiciones aéreas, lo cual estimula respuestas adaptativas altamente específicas (e.g. alta tolerancia a la desecación, desarrollo de vías de captación de oxígeno alternativas), las que han sido estudiadas principalmente en organismos sésiles. En este trabajo se analizó la respuesta fisiológica a la exposición aérea en las concentraciones de calcio y presión parcial de $\mathrm{CO}_{2}$ de la hemolinfa de la jaiba intermareal Petrolisthes laevigatus. Se sometió experimentalmente a individuos de esta especie a distintos periodos de exposición aérea $(0,6,15,60,240$ y $360 \mathrm{~min})$. Se evaluaron las variaciones de $\mathrm{pH}$, concentración de calcio $\left(\mathrm{Ca}^{2+}\right)$ hemolinfáticos y del exoesqueleto, ión bicarbonato $\left(\mathrm{HCO}_{3}^{-}\right)$presión parcial de $\mathrm{CO}_{2}\left(\mathrm{PCO}_{2}\right)$ de la hemolinfa. Los resultados indican una disminución de la concentración de calcio $\left[\mathrm{Ca}^{2+}\right]$ del exoesqueleto a medida que aumenta el tiempo de exposición aérea, lo cual fue seguido con un aumento de la concentración de $\left[\mathrm{Ca}^{2+}\right]$ en la hemolinfa. Estos resultados pueden indicar una disolución del carbonato de calcio del exoesqueleto que le permitiría a esta especie una regulación de equilibrio ácido-base durante periodos de emersión. El menor valor de $\mathrm{pH}$, y los valores mayores de concentración de $\mathrm{HCO}_{3}{ }^{-}$y $\mathrm{PCO}_{2}$ se encontraron en los organismos sumergidos (tiempo 0). Esto indicaría una alcalosis respiratoria durante la exposición aérea, lo que resultaría en una menor capacidad para mantener una respiración durante periodos de inmersión para esta especie.

Palabras clave: Jaiba, homeostasis, adaptación fisiológica, respiración antrópicos (e.g. contaminación) (Fernández et al. 2000).

Los organismos intermareales pueden estar expuestos al ambiente aéreo más del 50\% de sus vidas (Stillman \& Somero 1996). La facultad de soportar largos períodos de hipoxia depende en gran medida de su capacidad de utilizar el oxígeno atmosférico, de una alta tolerancia tanto a la desecación como a la exposición a altas temperaturas (Davenport 1972, Pellegrino 1984, O’Mahoney \& Full 1984, Jensen \& Armstrong 1991, Luquet \& Ansaldo 1997, 
Morris 2002, Gannon \& Henry 2004). Estas capacidades han sido preferentemente estudiadas en organismos sésiles tales como cirripedios y algas (Martin \& Bridges 1999, Dahlhoff et al. 2002, Yan \& Tanaka 2002), pero escasamente estudiadas en organismos móviles, como peces y crustáceos. En estos organismos, la exposición al medio aéreo provoca el colapso de las branquias, imposibilitando la captación de $\mathrm{O}_{2} \mathrm{y}$ generando una alteración de la homeostasis interna del organismo, causada por el cambio en el $\mathrm{pH}$ de la hemolinfa dado por la retención de $\mathrm{CO}_{2}$ (Johnson \& Uglow 1985, Varley \& Greenaway 1992, Luquet \& Ansaldo 1997).

La regulación del $\mathrm{pH}$ del medio interno es efectuada principalmente por acción de la enzima anhidrasa carbónica que participa en la conversión de $\mathrm{CO}_{2}$ y agua a bicarbonato y/o ácido carbónico (Randall et al. 2001). Además, en crustáceos ocurre un intercambio branquial de iones con el exterior $\left(\mathrm{Cl}^{-}\right.$por $\mathrm{HCO}_{3}^{-} \mathrm{y} \mathrm{Na}^{+}$por $\left.\mathrm{H}^{+}\right)$, que participan activamente en la regulación ácido-base (Henry et al. 1981, Henry \& Wheatly 1992, Luquet \& Ansaldo 1997, Henry 2001). Dado que en crustáceos expuestos al aire, la toma de $\mathrm{HCO}_{3}{ }^{-}$desde el agua es nula, la fuente de esta molécula debería ser otra; se ha sugerido que el $\mathrm{CaCO}_{3}$ del exoesqueleto podría ser movilizado durante periodos de acidosis y servir como mecanismo compensatorio (Henry et al. 1981). $\mathrm{El} \mathrm{CaCO}_{3}$ es altamente soluble y sensible a los cambios de $\mathrm{pH}$ dentro de rangos fisiológicos. Estas reservas de $\mathrm{CaCO}_{3}$ serían movilizadas durante acidosis causada por hipercapnia (aumento de la presión parcial de $\mathrm{CO}_{2}$ en la hemolinfa) en crustáceos terrestres durante períodos de exposición aérea, periodo en el cual la regulación del equilibrio ácido-base a nivel branquial ya no es posible (Henry et al. 1981, Wheatly \& Henry 1992).

La disolución de $\mathrm{CaCO}_{3}$ del exoesqueleto puede ser cuantificada a través de un cambio en la concentración de $\mathrm{Ca}^{2+}$ en la hemolinfa, al menos en condiciones experimentales donde los crustáceos son sometidos a exposiciones aéreas (Luquet \& Ansaldo 1997). Este mecanismo es de gran importancia en el proceso de adaptación de los organismos a nuevos ambientes, ya que de una regulación eficiente del medio interno dependen todas las funciones fisiológicas que permiten la supervivencia de los individuos (Henry 1994, Magnum 1994, Morris 2002, Farrelly \& Greenaway 2005).

Los crustáceos anomuros de la familia Porcellanidae son comunes en el intermareal rocoso (Stillman 2007). El gran número de especies del género Petrolisthes, su amplia distribución tanto geográfica, a través de aguas temperadas y tropicales, como en la franja intermareal (Carvacho 1980), hacen de este género un grupo ideal para el estudio de los mecanismos fisiológicos que permiten a los organismos intermareales adecuarse a las fluctuaciones de su ambiente (Stillman 2007).

Petrolisthes laevigatus (Guérin, 1835) es una especie común en la zona intermareal de las costas rocosas de Chile por lo que se encuentra sometido constantemente a periodos de emersión (Viviani 1969). Habita desde Perú $\left(12^{\circ} \mathrm{S}\right)$ hasta el sur de Chile $\left(46^{\circ} \mathrm{S}\right)$ (Retamal 1981). La exposición aérea inhibiría el intercambio de iones con el medio externo, lo que limitaría la capacidad de mantención del equilibrio interno, y por ende su supervivencia en el medio aéreo, produciéndose una acidosis por acumulación de $\mathrm{CO}_{2}$. Lo anterior podría ser moderado mediante la utilización de componentes estructurales como fuente de iones. En este trabajo se sometió a prueba esta capacidad por medio del estudio de las variaciones del equilibrio ácido-base de la hemolinfa a través del monitoreo de los cambios de $\mathrm{pH}$, concentraciones de $\mathrm{HCO}_{3}^{-}, \mathrm{CO}_{2} \mathrm{y} \mathrm{Ca}^{2+}$ en la hemolinfa y $\mathrm{Ca}^{2+}$ en el exoesqueleto durante la exposición aérea producto del ciclo mareal al que está sometido Petrolisthes laevigatus.

\section{Material y métodos}

En otoño e invierno 2003, se recolectaron manualmente 100 ejemplares machos de Petrolisthes laevigatus, con un rango de tallas entre $17-20 \mathrm{~mm}$ de ancho cefalotoráxico, del intermareal en la bahía San Vicente $\left(36^{\circ} 45^{\prime} \mathrm{S}-70^{\circ} 10^{\prime} \mathrm{W}\right)$, zona centro sur de Chile. Se transportaron en contenedores aislados térmicamente al Laboratorio de Fisiología Animal de la Universidad Católica de la Santísima Concepción, donde los animales fueron aclimatados individualmente en una cámara refrigerada, en recipientes con agua de mar a $13 \pm 0,5^{\circ} \mathrm{C}$, salinidad de 30 ups, por un lapso de $72 \mathrm{~h}$ (Chen \& Chia 1997, Lucu \& Devescovi 1999), antes del comienzo del periodo experimental.

\section{Equilibrio ácido-base}

Para evaluar los mecanismos de regulación ácido-base durante periodos de emersión, los individuos de esta especie fueron sometidos a diferentes tiempos de exposición aérea, 0 (organismos completamente sumergidos), 6, 15, 60, 120, 240, y 360 min (Luquet \& Ansaldo 1997). Diez ejemplares por tratamiento fueron colocados en contenedores individuales de $250 \mathrm{~mL}$, los cuales en su interior contenían arena humedecida con agua de mar para evitar la desecación. La arena fue previamente esterilizada dentro de un autoclave a $120^{\circ} \mathrm{C}$ durante $3 \mathrm{~h}$ (Greipsson \& El-Mayas 2000) y el agua filtrada a 0,45 $\mu \mathrm{m}$. Los recipientes fueron posteriormente colocados al 
interior de una cámara refrigerada por el tiempo de exposición definido a la misma temperatura de aclimatación.

Al finalizar cada periodo experimental, se tomaron muestras individuales de $1 \mathrm{~mL}$ de hemolinfa de cada individuo mediante punción en las quelas con jeringas de tuberculina. Inmediatamente después de su extracción las muestras fueron procesadas, midiéndose los siguientes parámetros: $\mathrm{pH}$ con un pHímetro HANNA modelo 1332; concentración de $\mathrm{HCO}_{3}^{-}$, según el método de Seagal modificado para la determinación titrimétrica de $\mathrm{HCO}_{3}{ }^{-}$ (Henry et al. 1980); presión parcial de $\mathrm{CO}_{2}$, mediante la ecuación de Henderson \& Hasselbalch, procedimiento señalado en la literatura (Henry et al. 1981, Waldron et al. 1986, Byrne \& McMahon 1991, Varley \& Greenaway $1992), \mathrm{pK}=\mathrm{pH}-\ln \left(\left[\mathrm{HCO}_{3}^{-}\right] / \alpha_{\mathrm{CO}_{2}}{ }^{2} \mathrm{PCO}_{2}\right)$. Se utilizaron los valores $\mathrm{HCO}_{3}^{-} \mathrm{y}$ pH obtenidos en los experimentos anteriores, y los valores de $\alpha \mathrm{CO}_{2}=0,0409$ y $\mathrm{pK}=6,073$ (Henry et al. 1981, Waldron et al. 1986); concentración de $\mathrm{Ca}^{2+}$, según el método de Moorehead \& Biggs (1974) y concentración de $\mathrm{Ca}^{2+}$ en exosqueleto, para lo cual se extrajeron $0,5 \mathrm{~g}$ del caparazón del cefalotórax de cada individuo, luego se agregaron $2,0 \mathrm{~mL}$ de $\mathrm{HCl} 0,1 \mathrm{M}$ a cada muestra para triturarlo con un mortero de porcelana. De este homogeneizado se extrajeron $10 \mu \mathrm{L}$ para medir las absorbancias a $570 \mathrm{~nm}$, según el método de Moorehead \& Biggs (1974).

\section{Análisis estadísticos}

Las diferencias estadísticas para cada variable fisiológica medida a diferentes tiempos de exposición aérea fueron analizadas mediante un ANDEVA de una vía (Zar 1996) seguida de una prueba a-posteriori de Tukey. Para verificar la homogeneidad de varianza de los datos se utilizó la prueba de Cochran (Zar 1996). Los datos fueron previamente transformados a $\log (\mathrm{x})$.

Para determinar el grado de relación entre las variables medidas se utilizó un análisis de correlación de Pearson (Zar 1996). Los datos se presentan como promedio más error estándar.

\section{Resultados}

El tamaño promedio de los organismos utilizados fue $19,45 \pm 0,64 \mathrm{~mm}$ de longitud cefalotoráxica y masa corporal de $8,27 \pm 0,09 \mathrm{~g}$ (Tabla 1A). No hubo diferencias significativas entre los diferentes grupos experimentales (ANDEVA: $\left.\mathrm{F}_{(6,63)}=0,58 ; P=0,74\right)$.

$\mathrm{El} \mathrm{pH}$ fue significativamente distinto entre los diferentes tiempos de exposición aérea. Se observó el menor valor de $\mathrm{pH}(7,55 \pm 0,03)$ en los organismos
Tabla 1

Resumen de los resultados de las ANDEVAs de una vía para la relación entre las concentraciones medidas en hemolinfa y exoesqueleto de Petrolisthes laevigatus a diferentes tiempos de exposición aérea $(G L=$ Grados de libertad, $C M=$ Cuadrados medios, $P=$ Probabilidad)

Summary of results from one way ANOVA about hemolymphatic and exosqueleton concentrations measured over several aerial exposure times of Petrolisthes laevigatus $(\mathrm{GL}=$ Freedom degrees, $\mathrm{CM}=$ Mean square, $P=$ Probability value)

\begin{tabular}{llrrrc}
\hline Variable & & DF & CM & F & $P$ \\
\hline a) Tamaño Corporal & Efecto & 6 & 9,89 & 0,58 & 0,74 \\
& Error & 63 & 1,71 & & \\
b) $\mathrm{pH}$ Hemolinfa & Efecto & 6 & 0,24 & 26,86 & $<0,01$ \\
& Error & 63 & 0,00 & & \\
c) $\left[\mathrm{HCO}_{3}{ }^{-}\right]$Hemolinfa & Efecto & 6 & 178,29 & 14,22 & $<0,01$ \\
& Error & 63 & 12,53 & & \\
d) $\mathrm{PCO}_{2}$ Hemolinfa & Efecto & 6 & 76,11 & 18,84 & $<0,01$ \\
& Error & 63 & 4,04 & & \\
f) $\left[\mathrm{Ca}^{2+}\right]$ Hemolinfa & Efecto & 6 & 9,90 & 19,83 & $<0,01$ \\
& Error & 56 & 0,49 & & \\
e) $\left[\mathrm{Ca}^{2+}\right]$ Exoesqueleto & Efecto & 6 & 0,07 & 13,39 & $<0,01$ \\
& Error & 63 & 0,05 & & \\
\hline
\end{tabular}

sumergidos (tiempo 0) (Fig. 1A), mientras que el mayor valor de $\mathrm{pH}$ se observó a los 15 minutos de exposición aérea con un valor promedio de 7,95 $\pm 0,02$. (ANDEVA $\left.\mathrm{F}_{(6,63)}=26,86 ; P<0,001\right)$ (Tabla 1B) (Tukey a-posteriori $P<0,05)$.

La concentración de $\mathrm{HCO}_{3}^{-}$fue significativamente distinta entre los tratamientos. La mayor concentración de $\mathrm{HCO}_{3}{ }^{-}$se observó a los 0 minutos de exposición aérea, con un valor promedio de $28,25 \pm 0,61 \mathrm{mmol} \mathrm{L}^{-1}$ (Fig. 1B). La menor concentración de $\mathrm{HCO}_{3}$ se observó a los 6 minutos de exposición, con un valor de 15,00 $\pm 1,30 \mathrm{mmol}$ $\mathrm{L}^{-1}\left(\right.$ ANDEVA $\left._{(6,63)}=14,22 ; P<0,001\right)$ (Tukey $a-$ posteriori $P<0,05$ ) (Tabla $1 \mathrm{C}$ ).

La mayor presión parcial de $\mathrm{PCO}_{2}$ en la hemolinfa de $P$. laevigatus fue observada en los organismos no expuestos (tiempo 0 ), con un valor promedio $15,77 \pm 0,71$ $\mathrm{mm} \mathrm{Hg}$ (Fig. 1C). $\mathrm{La} \mathrm{PCO}_{2}$ fue significativamente distinta entre los organismos en inmersión y los expuestos a condiciones aéreas. El valor menor de $\mathrm{PCO}_{2}$ se observó a los 6 minutos de exposición aérea, con promedio de 7,97

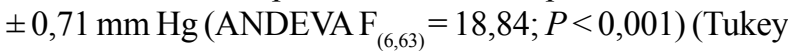
a-posteriori $P<0,05)$ (Tabla 1D).

La concentración de $\mathrm{Ca}^{2+}$ en la hemolinfa aumentó con el tiempo de exposición aérea, con un promedio mínimo de 14,34 $\pm 0,13 \mathrm{mmol} \mathrm{L}^{-1}$ a los 6 min (Fig. 1D). El mayor valor fue observado a los 60 min de exposición, 

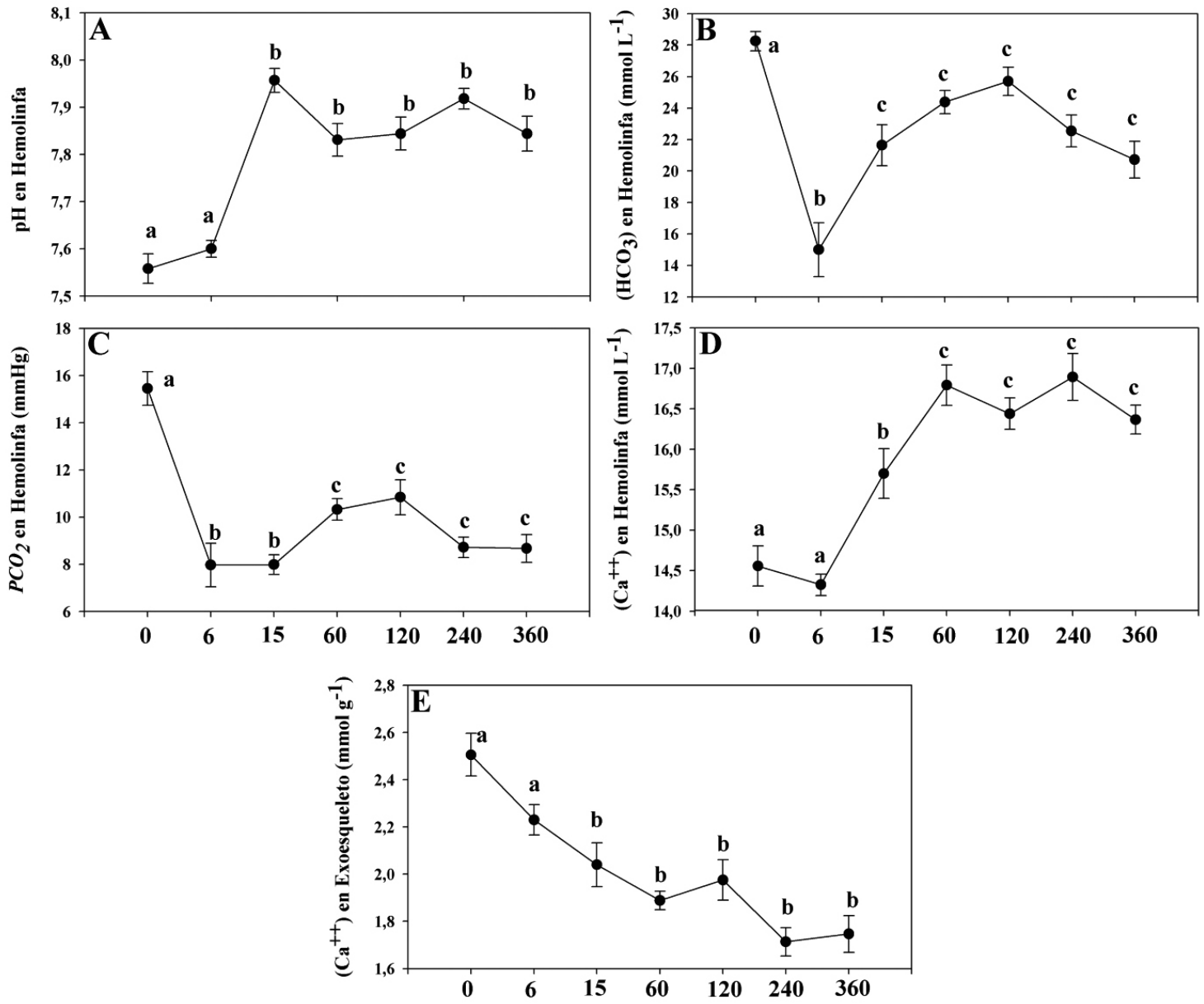

Tiempo de exposición aérea (min)

Figura 1

Concentraciones medidas a distintos tiempos de exposición aérea en el crustáceo intermareal Petrolisthes laevigatus ( \pm EE). $\mathrm{A}=$ Variaciones en pH hemolinfático; $\mathrm{B}=$ Concentración hemolinfática de $\mathrm{H} \mathrm{CO}_{3} ; \mathrm{C}=$ Presión parcial en la hemolinfa de $\mathrm{CO}_{2}$; $\mathrm{D}=$ Concentración hemolinfática de Calcio; $\mathbf{E}=$ Concentración de calcio en exoesqueleto. Letras diferentes indican diferencias significativas entre los tratamientos (Tukey a-posteriori, $P<0,05$ )

Concentrations measured in the intertidal crab Petrolisthes laevigatus over several times of aerial exposure ( $\mathrm{x} \pm \mathrm{EE})$. $\mathrm{A}=\mathrm{Variations}$ in hemolymphatic $\mathrm{pH} ; \mathrm{B}=\mathrm{HCO}_{3}$ hemolymphatic concentrations; $\mathrm{C}=$ Hemolymphatic $\mathrm{CO}_{2}$ partial pressure; $\mathrm{D}=\mathrm{Calcium}$ hemolymphatic concentrations; $\mathrm{E}=$ Calcium exoesqueleton concentration. Different letters denote significant differences among treatments (Tukey a-posteriori, $P<0.05$ )

con un promedio de $16,79 \pm 0,25 \mathrm{mmol} \mathrm{L}^{-1}$. (ANDEVA $\left.\mathrm{F}_{(6,56)}=19,83 ; P<0,001\right)($ Tabla $1 \mathrm{~F})$ (Tukey a-posteriori $P<0,05)$.

La concentración de $\mathrm{Ca}^{2+}$ en el exoesqueleto fue significativamente distinta entre tratamientos. Se observó que la concentración de $\mathrm{Ca}^{2+}$ disminuyó a través del tiempo de exposición aérea. En organismos sumergidos se midió el mayor valor $\left(2,50 \pm 0,09 \mathrm{mmol} \mathrm{g}^{-1}\right)$, el valor más bajo se observó a los 240 minutos $(1,71 \pm 0,05 \mathrm{mmol}$ $\left.\mathrm{g}^{-1}\right)$ (Fig. 1E), (ANDEVAF $\left.{ }_{(6,63)}=13,39 ; P<0,001\right)$ (Tukey a-posteriori $P<0,05$ ) (Tabla $1 \mathrm{E}$ ).

\section{Análisis de correlación}

Se encontraron correlaciones positivas y significativas entre: la concentración de $\mathrm{Ca}^{2+}$ y el $\mathrm{pH}$ en hemolinfa (rs $=0,52, P<0,01)$ (Fig. 2C) (Tabla 1D), la concentración de $\mathrm{HCO}_{3}^{-}$y la $\mathrm{PCO}_{2}$ en hemolinfa (rs $=0,88, P<0,01$ ) (Fig. 2D) (Tabla 2E), y la $\mathrm{PCO}_{2}$ de la hemolinfa y la 

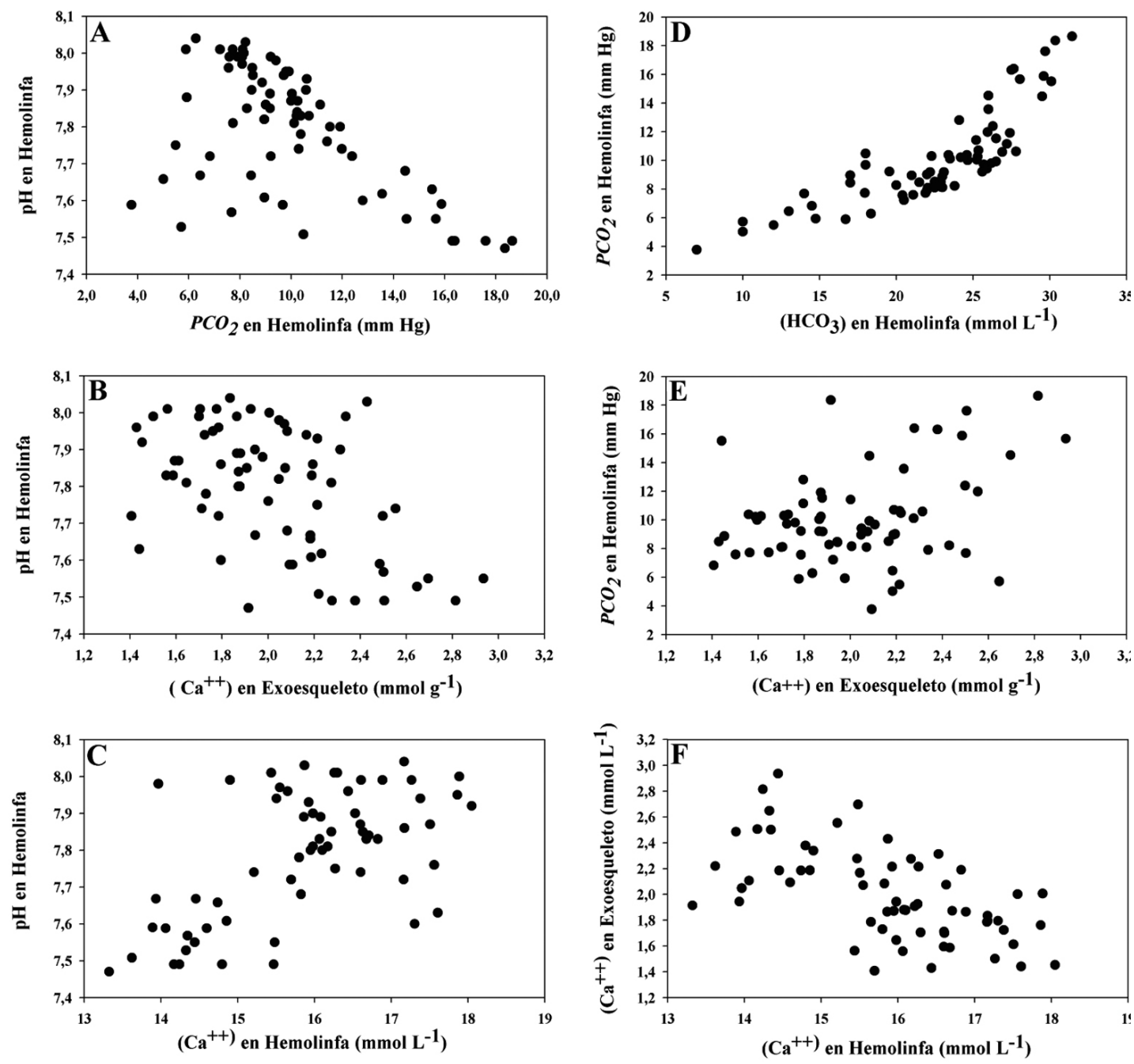

Figura 2

Correlaciones significativas entre variables hemolinfáticas medidas en los diferentes tiempos de exposición aérea de Petrolisthes laevigatus

Correlations between hemolymphatics variables measured over different aerial exposition times in Petrolisthes laevigatus

concentración de $\mathrm{Ca}^{2+}$ en el exoesqueleto ( $\mathrm{rs}=0,25, P<$ 0,05) (Fig. 2E) (Tabla 2H). Por otra parte se observaron relaciones negativas y significativas entre: el pH y la $\mathrm{PCO}_{2}$ en la hemolinfa ( $\mathrm{rs}=-0,49, P<0,01)$ (Fig. 2A) (Tabla 2B), el pH de la hemolinfa y la concentración de $\mathrm{Ca}^{2+}$ en exoesqueleto ( $\mathrm{rs}=0,46, P<0,01$ ) (Fig. 2B) (Tabla 2C), y la concentración de $\mathrm{Ca}^{2+}$ en exoesqueleto y el $\mathrm{Ca}^{2+}$ en hemolinfa ( $\mathrm{rs}=-0,06, P<0,05)($ Fig. 2F) (Tabla 2J).

\section{Discusión}

Las respuestas hemolinfáticas observadas en Petrolisthes laevigatus no son las clásicamente descritas en crustáceos acuáticos, ya que en estos organismos la inmersión está asociada principalmente a un aumento del $\mathrm{pH}$ de la hemolinfa y a una disminución de la presión parcial de $\mathrm{CO}_{2}$ (alcalosis respiratoria) (Greenaway 2003). En este estudio se observó que los individuos en inmersión presentaron una respuesta contraria, con una disminución del $\mathrm{pH}$ hemolinfático y una aumento de la presión parcial de $\mathrm{CO}_{2}$ (hipercapnia), en comparación con los organismos en emersión (Fig. 1C); esto indicaría una menor capacidad de esta especie para respirar bajo el agua en comparación a periodos de emersión (Henry et al. 1981). Una respuesta similar se observó en el crustáceo anfibio Cardisoma carnifex, que durante periodos de inmersión sufre un aumento de la $\mathrm{PCO}_{2}$ y un descenso del pH en comparación 


\section{Tabla 2}

Valores de correlación de Spearman entre las concentraciones medidas en Petrolisthes laevigatus ( $\mathrm{N}=$ Tamaño muestreal, $\boldsymbol{P}=$ Probabilidad $)($ Exo $=$ Exoesqueleto, $\mathrm{Hem}=$ Hemolinfa $)$

Spearman correlation values between measured concentrations in Petrolisthes laevigatus $(\mathrm{N}=$ Simple size, $P=$ Probability value $)($ Exo $=$ Exoskeleton, Hem $=$ Hemolymph $)$

\begin{tabular}{|c|c|c|c|c|}
\hline Variables & $\mathrm{N}$ & Spearman(rs) & $\mathrm{t}(\mathrm{N}-2)$ & $P$ \\
\hline a) $\mathrm{pH}$ y $\left[\mathrm{HCO}_{3}^{-}\right]$ & 70 & $-0,14$ & $-1,24$ & 0,22 \\
\hline b) $\mathrm{pH}$ y $\mathrm{PCO}_{2}$ & 70 & $-0,49$ & $-4,63$ & $<0,01$ \\
\hline c) $\mathrm{pH}$ y $\left[\mathrm{Ca}^{2+}\right]$ Exo & 70 & $-0,46$ & $-4,22$ & $<0,01$ \\
\hline d) $\mathrm{pH} y\left[\mathrm{Ca}^{2+}\right] \mathrm{Hem}$ & 63 & 0,52 & 4,73 & $<0,01$ \\
\hline e) $\left[\mathrm{HCO}_{3}^{-}\right]$y $\mathrm{PCO}_{2}$ & 70 & 0,88 & 15,10 & $<0,01$ \\
\hline f) $\left[\mathrm{HCO}_{3}^{-}\right]$y $\left[\mathrm{Ca}^{2+}\right]$ Exo & 70 & 0,15 & 1,28 & 0,20 \\
\hline g) $\left[\mathrm{HCO}_{3}^{-}\right]$y $\left[\mathrm{Ca}^{2+}\right] \mathrm{Hem}$ & 63 & 0,09 & 0,74 & 0,46 \\
\hline h) $\mathrm{PCO}_{2} \mathrm{y}\left[\mathrm{Ca}^{2+}\right]$ Exo & 70 & 0,25 & 2,12 & $<0,05$ \\
\hline i) $\mathrm{PCO}_{2} \mathrm{y}\left[\mathrm{Ca}^{2+}\right] \mathrm{Hem}$ & 63 & $-0,04$ & $-0,33$ & 0,74 \\
\hline j) $\left[\mathrm{Ca}^{2+}\right]$ Exo. y $\left[\mathrm{Ca}^{2+}\right] \mathrm{Hem}$ & 63 & $-0,06$ & $-5,85$ & $<0,01$ \\
\hline
\end{tabular}

a la emersión, con un valor similar al observado en $P$. laevigatus (Morris \& Adamezewska 1996).

En la mayor parte de los organismos, la disminución del $\mathrm{pH}$ y el aumento de la $\mathrm{PCO}_{2}$ ocurre durante periodos de disminución de la captación de $\mathrm{O}_{2}$ y de retención de $\mathrm{CO}_{2}$ (Randall et al. 2001). En P. laevigatus, la combinación de bajo $\mathrm{pH}$, alta $\mathrm{PCO}_{2}$ y alta concentración de $\mathrm{HCO}_{3}^{-}$(Fig. 1) se asocia a un estado de acidosis respiratoria, la cual podría ser causada por una disminución en el área superficial branquial en comparación a otras especies de crustáceos, lo que limitaría su capacidad de mantener una efectiva respiración en el agua, esta podría ser una ventaja en emersión, ya que disminuye la pérdida de agua por evaporación (Gray 1957, Hawkins \& Jones 1982, Henry et al. 1990, Henry 1991, Greenaway 2003). Otros trabajos con esta misma especie indican que $P$. laevigatus en inmersión presenta una alta actividad de la enzima lactato deshidrogenasa (LDH) (Cáceres, datos no publicados), lo cual indica el uso de metabolismo anaeróbico en estas condiciones, lo que trae como consecuencia una acidificación en la hemolinfa (Varley \& Greenaway 1992).

Durante la exposición aérea, $P$ laevigatus presentó a los 60 minutos una regulación del $\mathrm{pH}$, similar a lo que se ha descrito en otras especies como Chasmagnathus granulata (Luquet \& Ansaldo 1997), la cual se mantuvo durante todo el período de exposición (Fig. 1A). En este tipo de crustáceos la regulación de $\mathrm{pH}$ parece depender de la SID ('strong ion difference'), que es la diferencia entre la suma de cationes fuertes y la suma de aniones fuertes, que se regula a través de dos diferentes mecanismos, dependiendo de la disponibilidad de agua; así, en crustáceos acuáticos estaría siendo regulada a través del intercambio iónico por medio de las branquias, mientras que crustáceos terrestres y semiterrestres lo estaría haciendo a través de la disolución de $\mathrm{CaCO}_{3}$ del exoesqueleto, lo cual genera $\mathrm{Ca}^{2+} \mathrm{y} \mathrm{HCO}_{3}^{-}$(Henry et al. 1981, Truchot 1990, Luquet \& Ansaldo 1997). El aumento de las concentraciones de calcio en la hemolinfa durante la emersión (Fig. 2F) indica claramente que esta especie utiliza este mecanismo para regular el $\mathrm{pH}$ hemolinfático (Fig. 1A).

La mayor concentración de $\mathrm{HCO}_{3}^{-}$se observó en los individuos en inmersión (Fig. 2D), asociado a una alta $\mathrm{PCO}_{2}$ y un bajo valor de $\mathrm{pH}$ hemolinfático. En estas condiciones, la captación de $\mathrm{HCO}_{3}^{-}$puede ser a través de las branquias directamente desde el agua. Esto se ha descrito en otros crustáceos como Callinectes sapidus, en el cual la compensación de una acidosis fue acompañada por la captación de $\mathrm{HCO}_{3}{ }^{-}$(Henry \& Wheatly 1992, Whiteley et al. 2001).

A pocos minutos de exposición aérea se produjo un fuerte descenso en la concentración de $\mathrm{HCO}_{3}^{-}$en la hemolinfa de P. laevigatus (Fig 1B), respuesta fisiológica previamente observada en otras especies de crustáceos como Carcinus maenas y Pacifasticus leniusculus (Whiteley et al. 2001). La disminución en la concentración de $\mathrm{HCO}_{3}^{-}$en la hemolinfa durante la recuperación de un estado de hipercapnia puede ser atribuida e la excreción de base equivalentes (Whiteley et al. 2001). Posteriormente, entre los 15 y 60 minutos se vuelve a producir un aumento en la concentración de $\mathrm{HCO}_{3}{ }^{-}$; en este punto, la toma de $\mathrm{HCO}_{3}{ }^{-}$dependería de la capacidad para retener agua como se ha observado en otros crustáceos como Chasmagnathus granulatus (Halperin et al. 2000), o de la capacidad de disolver el $\mathrm{CaCO}_{3}$ en $\mathrm{Ca}^{2+}+\mathrm{HCO}_{3}^{-}$como se ha sugerido en la literatura (Henry et al. 1981).

Al aumentar el tiempo de exposición al aire $P$. laevigatus respondió con un aumento de la concentración de $\mathrm{Ca}^{2+}$ de la hemolinfa (Fig 1D), lo que se correlacionó negativamente con la disminución de la concentración de $\mathrm{Ca}^{2+}$ del exoesqueleto y positivamente con el $\mathrm{pH}$. Este incremento se mantuvo hasta la hora de exposición aérea, tiempo en el cual se produjo la estabilización del pH (Fig 1A). El aumento de la acidez como respuesta a la exposición aérea ha sido observada en algunos crustáceos terrestres como Gecarcinus lateralis pero no en otros completamente acuáticos como Callinectes sapidus (Henry et al. 1981). Esto podría indicar una mayor 
capacidad de esta especie de regular su respiración en un medio aéreo en comparación con el acuático.

La disolución del $\mathrm{CaCO}_{3}$ del exoesqueleto estaría jugando un rol importante en la regulación del equilibrio ácido-base a través de la entrega de $\mathrm{HCO}_{3}$ - como ha sido ampliamente sugerido no sólo para crustáceos (Henry et al. 1981, Innes et al. 1986), sino también para moluscos (Bryne \& McMahon 1991). Además, la movilización de $\mathrm{CaCO}_{3}$ del exoesqueleto es un indicador del grado de independencia de sistemas acuáticos, ya que el incremento en la concentración de $\mathrm{Ca}^{2+}$ en la hemolinfa ocurre solamente en crustáceos terrestres, en los cuales la regulación ácido base a nivel branquial no resulta una opción viable (Henry et al. 1981, Innes et al. 1986).

Al parecer, Petrolisthes laevigatus tendría una alta capacidad de respiración aérea, lo cual le facilitaría soportar la emersión en cada ciclo mareal. Esta habilidad puede estar relacionada con el desarrollo de dos estructuras anexas que le permitirían la captación de oxígeno del aire: (a) segmentos descalcificados, altamente vascularizados en la cara anterior del mesopodito de los apéndices locomotores; dentro de los porcelánidos, esta especie es la que presente las membranas de mayor área (Stillman 2000), y (b) segmentos descalcificados en la zona lateral del cefalotórax, la funcionalidad de estas estructuras respiratorias ha sido descrita en especies como Chasmagnathus granulatus (Farrelly \& Greenaway 1994, Halperin et al. 2000), pero no aún en porcelánidos.

La habilidad de regular el equilibrio ácido-base durante la emersión de Petrolisthes laevigatus es una gran ventaja que le permitiría habitar la zona alta del intermareal, por lo que las prolongadas exposiciones a condiciones aéreas no significaría un estrés fisiológico desde el punto de vista respiratorio.

\section{Agradecimientos}

Los autores agradecen al Sr. Moisés Vargas por su ayuda en terreno y en laboratorio. Se agradece a los revisores anónimos por sus comentarios y sugerencias. Este trabajo forma parte de la Tesis de Grado de M. Lagos en la carrera de Biología Marina de la UCSC.

\section{Literatura citada}

Byrne R \& B Mcmahon. 1991. Acid-base and ionic regulation, during and following emersion, in the freshwater bivalve, Anodonta grandis (Bivalvia: Unionidae). The Biological Bulletin 181: 289-297.

Carvacho A. 1980. Los porcelánidos del pacifico americano: un análisis biogeográfico (Crustacea: Decapoda). Anales del Centro del Mar y de Limnología, Universidad Nacional Autónoma de México 7(2): 249-258.
Chen J \& P Chia. 1997. Osmotic and ionic concentrations of Scylla serrata (Forskål) subjected to different salinity levels. Comparative Biochemistry and Physiology Part A: Physiology 117(2): 239-244.

Dahlhoff E, J Stillman \& B Menge. 2002. Physiological community ecology: Variation in metabolic activity of ecologically important rocky intertidal invertebrates along enviromental gradients. Integrative and Comparative Biology 42: $862-871$.

Davenport J. 1972. Salinity tolerance and preference in the Porcelain crabs Porcellana platycheles and Porcellana longicornis. Marine Behaviour and Physiology 1: 123-138.

Farrelly C \& P Greenaway. 1994. Gas exchange through the lungs and gills in air breathing crabs. Journal of Experimental Biology 187: 113-130.

Farrelly C \& P Greenaway. 2005. The morphology and vasculature of the respiratory organs of terrestrial hermit crabs (Coenobita and Birgus): gills, branchiostegal lungs and abdominal lungs. Arthropod Structure \& Development 34(1): 63-87.

Fernández M, E Jaramillo, P Marquet, C Moreno, S Navarrete, P Ojeda, C Valdovinos \& J Vásquez. 2000. Diversidad, dinámica y biogeografía del ecosistema costero bentónico de Chile: revisión y bases para conservación marina. Revista Chilena de Historia Natural 73(4): 797-830.

Gannon A \& R Henry. 2004. Oxygen and carbon dioxide sensitivity of ventilation in amphibious crabs, Cardisoma guanhumi, breathing air and water. Comparative Biochemistry and Physiology - Part A: Molecular and Integrative Physiology 138(1): 111-117.

Greenaway P. 2003. Terrestrial adaptation in the Anomura (Crustacea: Decapoda). Memoirs of Museum Victoria 60(1): 13-26.

Greipsson S \& H El-Maya. 2000. Arbuscular mycorrhizae of Leymus arenarius on coastal sands and reclamation sites in Iceland and response to inoculation. Restoration Ecology 8: 144-150.

Gray I. 1957. A comparative study of the gill area of crabs. The Biological Bulletin 112: 34-42.

Halperin J, MAnsaldo, GPellerano \& C Luquet. 2000. Bimodal breathing in the estuarine crab Chasmagnathus granulatus (Dana 1851), physiological and morfological studies. Comparative Biochemistry and Physiology B126: 341-349.

Hawkins A \& M Jones. 1982. Gill area and ventilation in two mud crabs, Helice crassa Dana (Grapsidae) and Macrophthalmus hirtipes (Jacquinot) (Ocypodidae), in relation to habitat. Journal of Experimental Biology and Ecology 60: 103-118.

Henry R. 1991. Branchial and branchiosteguite carbonic anhydrase in decapod crustaceans: The aquatic to terretrial transition. The Journal of Experimental Zoology 259: 294303.

Henry R. 1994. Morphological, behavioral and physiological characterization of bimodal breathing crustaceans. American Zoologist 34: 205-215. 
Henry R, D Cannon \& J Winkelman. 1980. Química clínica, bases y técnicas: Tomo 1, 819 pp. Editorial Jims, Barcelona.

Henry R, G Kormanik, N Smatresk \& J Cameron. 1981. The role of $\mathrm{CaCO}_{3}$ dissolution as a source of $\mathrm{HCO}_{3}^{-}$for the buffering of hypercapnic acidosis in the aquatic and terrestrial decapod crustaceans. The Journal of Experimental Biology 94: 269274.

Henry R, H Perry, C Trigg, H Handley \& A Krarup. 1990. Physiology of two species of deep water crabs, Chaceon fenneri and C. quinquedens: Gill morphology, a hemolymph ionic and nitrogen concentrations. Journal of Crustacean Biology 10: 375-381.

Henry R \& M Wheatly. 1992. Interaction of respiration, ion regulation, and acid-base balance in the everyday life of aquatic crustaceans. American Zoologist 32: 407-416.

Henry R. 2001. Enviromentally mediated carbonic anhydrase induction in the gills of euryaline crustaceans. The Journal of Experimental Biology 204: 991-1002

Innes A, M Foster, M Jones, I Marsden \& H Taylor. 1986. Bimodal respiration, water balance ad acid-base regulation in a high-shore crab, Cyclograpsus lavuaxi Milne Edwards. Journal of Experimental Marine Biology and Ecology 100: 127-145.

Jensen G \& D Armstrong. 1991. Intertidial zonation among congeners: factors regulating distribution of Porcelain crabs Petrolisthes spp. (Anomura: Porcellanidae). Marine Ecology Progress Series 73: 47-60.

Johnson I \& R Uglow. 1985. Some effects of aerial exposure on the respiratory physiology and blood chemistry of Carcinus maenas (L.) and Liocarcinus puber (L.). Journal of Experimental Marine Biology and Ecology 94: 151-165.

Lucu C \& M Devescovi. 1999. Osmoregulation and branchial $\mathrm{Na}^{+}, \mathrm{K}^{+}$-ATPase in the lobster Homarus gammarus acclimated to dilute seawater. Journal of Experimental Marine Biology and Ecology 234: 291-304.

Luquet C \& M Ansaldo. 1997. Acid-Base balance and ionic regulation during emersion in the estuarine intertidal crab Chasmagnathus granulata Dana (Decapoda Grapsidae). Comparative Biochemistry and Physiology A 117(3): 407-410.

Magnum C. 1994. Multiple sites of gas exchange. American Zoologist 34: 184-193.

Martin K \& C Bridges. 1999. Respiration in water and air. En: Horn M, K Martin \& M Chotwoski (eds), Intertidal fishes, life in two worlds, pp. 54-77. Academic Press, San Diego.

Moorehead W \& H Biggs. 1974. 2-Amino-2-methyl-1-propanol as the alkalizing agent in an improve continous-flow cresolphthalein complexo procedure for calcium in serum. Clinical Chemistry 20(11): 1458-1480.

Morris S. 2002. The ecophysiology of air-breathing in crabs with special reference to Gecarcoidea natalis. Comparative Biochemistry and Physiology B 131(4): 559-570.

Morris S \& A Adamezwska. 1996. Respiratory, acid-base and ion status during voluntary immersion of the air-breathing crab
Cardisoma carnifex assessed in situ. Journal of Experimental Biology and Ecology 206: 149-164.

O'Mahoney P \& R Full. 1984. Respiration of crabs in air and water. Comparative Biochemistry and Physiology A 79(2): 275282.

Pellegrino C. 1984. The role of desiccation pressures and surface area/volume relationships on seasonal zonation and size of four intertidal decapod crustacea from New Zealand: implication for adaptation to land. Crustaceana 47(3): 251268.

Randall D, W Burggren \& K French. 2001. Eckert, Animal Physiology: mechanisms and adaptations, $735 \mathrm{pp}$. WH Freeman Co, Nueva York

Retamal M. 1981. Catálogo ilustrado de los crustáceos decápodos de Chile. Gayana Zoología 44: 1-256.

Stillman J. 2000. Evolutionary history and adaptive significance of respiratory structures on the legs of intertidal Porcelain crabs. Physiological and Biochemical Zoology 73(1): 86-96.

Stillman J. 2007. Crabs. En: Denny M \& S Gaines (eds). Encyclopedia of Tidepools and Rocky Shores, pp. 164-168. University of California Press, Los Angeles.

Stillman J \& G Somero. 1996. Adaptations to temperature stress and aerial exposure in congeneric species of intertidal porcelain crabs (genus Petrolisthes): correlation of physiology, biochemistry and morphology with vertical distributions. The Journal of Experimental Biology 199: 1845-1855.

Truchot JP. 1990. Respiratory and ionic regulation in invertebrates exposed to both water and air. Annual Reviews of Physiology 52: 61-76.

Varley D \& P Greenaway. 1992. The effect of emersion on hemolimph acid-base balance and oxygen levels in Silla serrata Forskal (Brachyura: Portunidae). Journal of Experimental Biology and Ecology 163: 1-12.

Viviani C. 1969. Los Porcellanidos (Crustacea, Anomura) Chilenos. Sonderdruks aus Beitrage zur Neotropischen Fauna. Band 4(1): 4-56.

Waldron F, H Taylor \& M Foster. 1986. Acid-base disturbances following exercise in a high-shore crab, Cyclograpsus lavauxi. New Zealand Journal of Marine and Freshwater Research 20: 479-487.

Wheatly M \& R Henry. 1992. Extracellular and intracellular acidbase regulation in crustaceans. The Journal of Experimental Zoology 263: 127-142.

Whiteley N, J Scott, S Breeze \& L McCann. 2001. Effects of water salinity on acid-base balance in decapod crustaceans. The Journal of Experimental Biology 204: 1003-1001.

Yan J \& J Tanaka. 2002. Effect of desiccation on the photosynthesis of seaweeds from the intertidal zone in Honshu, Japan. Phycological Research 50(2): 145-153.

Zar J. 1996. Biostatistical analysis, 728 pp. Prentice-Hall, Englewood Cliffs. 